\title{
MPLS Based Mobility Framework in 4G Architectures
}

\author{
Iti Saha Misra \\ Dept. of Electronics and Telecommunication Engg. \\ Jadavpur University \\ Calcutta, India-700032 \\ +91-033-25556786 \\ itimisra@cal.vsnl.net.in \\ Anirban Banerjee \\ Indian Institute of Information Technology \\ Salt Lake City, Sector-5 \\ Calcutta, India-700106 \\ anir_iiit@yahoo.co.uk
}

\begin{abstract}
In this paper, an integration framework for 4Gnetwork architecture between UMTS-GPRS system and 802.11 WLAN systems using MPLS as the underlying technology is developed. In $4 \mathrm{G}$ networks, to suffice the needs of users with relatively low mobility but yet to provide a substantial amount of bandwidth in hot-spots, it becomes essential to integrate WLAN 802.11 systems with currently existing UMTS-GPRS systems. The integrated network can handle wider mobility of the mobile nodes (MN). Multiprotocol Level Switching (MPLS), a technology has in built features pivoting on QoS provides better efficiency in the integrated networks. In this paper it is shown that when MPLS is used to bridge the UMTS and 802.11 architectures, reduces location update of the $\mathrm{MN}$ with the core network $(\mathrm{CN})$. This is achieved by switching the parameters governing Forwad Equivalence Class (FEC) generation and redirection in such MPLS enabled architecture. An efficient mobility management scheme is discussed when MN moves from one domain to other. Simulation results for handoff delays and location update of an $\mathrm{MN}$ in the integrated architecture with MPLS show the better performance of the proposed MPLS based framework.
\end{abstract}

\section{INTRODUCTION}

The 3G cellular networks, e.g. UMTS are designed to provide users with voice and data services. Total cell capacity limits the per user data rate. Many times highspeed requirements are clustered in small pockets. These clusters are termed as hot-spots. Network operators would like to employ efficient solutions, which are easily integrated with their existing UMTS, based infrastructure. WLANs offer an attractive solution. 3G cellular accesses based on code division multiple access (CDMA) either wideband CDMA or cdma2000 can be used to satisfy users who have a larger need for mobility while 802.11 systems can be used to support users with much lesser coverage area requirements. It is in light of this, the next wave of technological advance is already under consideration i.e. 4G. Several International projects are already underway to ensure that services are suited to the characteristics of several different delivery mechanisms, from cable networks to GPRS networks, and the services can be delivered using the most appropriate of the range of networks available. In addition the dynamic allocation of available spectrum between different wireless networks is also under investigation. The challenge is to explore the design of such a transport infrastructure which will be able to take full advantage of IP based technologies achieving desired mobility between the various access techniques and at the same time provide the necessary capabilities in terms of QoS, robustness and manageability. The goals at the present stage regarding the development of mobile standards remains common (3GPP and 3GPP2) and include IP based multimedia services, IP based transport and the integration of IETF protocols for functions such as wide area mobility support (MIP), signaling (SIP) and authentication, authorization and accounting (AAA), it is popular to call any network that satisfies these criteria as an all-IP network.

We provide a brief overview of the underlying UMTS concepts in order to develop an all-IP network model and to explain the benefits of using technologies such as MPLS in the development of such a system. Very basic overviews of wireless protocols and technology concepts have been extracted from [1]. QoS provisioning in cellular networks is a major issue and research based on reservation of the needed resources using techniques based on mobility prediction by use of models based on second order Markov models has been discussed in [2]. Practical experiences with GEANT which is the panEuropean 10Gbs network interconnecting European NRENs and the implementation of the PremiumIP service based on the Diffserv EF PHB provides an insight into the practical implementation constraints in this area [3]. Services and network level management frameworks which play a major role in decision making in cellular networks as $3 \mathrm{G}$ have been formulated in a formal manner while cogently discussing problems such as SCNOS in [4]. Modelling of 4G networks using concepts of Hybrid Network components based on earlier established technologies to develop a framework for 4G [5] systems is core to the concepts discussed in this paper. A general perspective on $4 \mathrm{G}$ systems has been described in [6], [7]. An overview of $3 \mathrm{G}$ systems [8] and their issues related to micromobility have been discussed in [9], these deal with development of a new framework based on MPLS [10] to 
enhance the QoS framework in the proposed model. Suggestions for the development of lebel edge mobility agents (LEMA) to increase native IP penetration are core to the concepts explored in this paper. Destination based traditional IP routing has not been able to make full use of the multiple paths that may exist in a network operator's domain, MATE provides a flexible solution based on MPLS to elevate this underutilization scenario [11]. Related concepts for load balancing have been briefly described in [12]. A general overview of routing protocols, algorithms and the associated constraints in each of these schemes with relation to multicast services and it's QoS considerations are cogently described in [13]. A perspective on ATM in MPLS based core data networks offers a view into the integration of the services and the integration architectures of these two systems in [14]. A detailed overview to describe the internetworking between 802.11 WLAN systems and UMTS based systems has been provide in [15], development of criteria to select the integration points between two such networks with different radio frameworks is a major concept which is explored in this literature. We develop an efficient architecture for the integration of 802.11 WLAN and UMTS based networks and at the same time specify a framework within which we discuss the application of MPLS technology for QoS considerations. The architecture presented in this paper will focus on the core aspects of a $4 \mathrm{G}$ network such as all-IP architecture, global mobility, efficient handoff and QoS.

This paper is divided as follows. In section 1, a very brief concepts and motivation of the paper is given. Standard architectural details of UMTS-GPRS and 802.11 WLAN are provided in section 2 which ate the focal point of the integration mechanism. It also provides the deployment technique of MPLS in IP networks. The mobility and handoff management schemes for the proposed integrated architecture are given in section 3. Performance analysis in respect of handoff and signaling overhead is given in section 4. Finally, conclusion is given in section 5 .

\section{ARCHITECTURE}

\section{UMTS Overview [5], [9], [10], [15]}

$4 \mathrm{G}$ networks can be modeled as a basic UMTS domain within which there are islands of 802.11 WLANs to serve the hot-spots created by user requirements in particular locations. The basic UMTS network as shown in Fig. 1, provides packet data service (PS) and circuit switched voice service (CS). The GPRS network is integrated into UMTS to get the PS service. In UMTS, RNCs (Radio Network Controller) and Node Bs constitute the Radio Access Network (RAN) called UMTS RNS. Each Node B constitutes of a cluster of base stations or APs (Access Points), and number of Node Bs may be connected to a single RNC. The packet core network $(\mathrm{CN})$ comprises of SGSN (Serving GPRS Support Node) and GGSNs (Gateway GSN). In RAN, the RNC receives downlink packets from SGSN and converts them to radio frames before sending them over to the Node Bs. In the uplink direction the RNS receives the radio frames from the Node Bs and converts them to IP packets and dispatches them to the SGSN. The RNC manages the radio resources at the Node Bs and also is responsible for the maintenance of RABs (Radio Access Bearer) through them. The SGSN also maintains the mobility context of the UE (User Equipment) / MN and controls RAB setup through RNCs. Two GTP sessions (GPRS Tunneling Session) are created between SGSN-GGSN and SGSN-RNC for passage of IP packets. When multiple APs serving a MN have different controlling RNCs then one of the latter acts as the Serving-RNC for that host. It is this Serving-RNC that is responsible for frame selection among the multiple received copies of the same transport block. The MN attaches to the $\mathrm{CN}$ by GPRS-attach operation. This creates two GTP sessions between SGSN-GGSN and SGSNRNC. Upon GPRS attachment a mapping is created at the RNC between host ID and GTP session and RNC and SGSN. Another mapping is created at GGSN between hosts network IP address and SGSN. SGSN handles interRNC mobility and GGSN handles inter SGSN mobility.

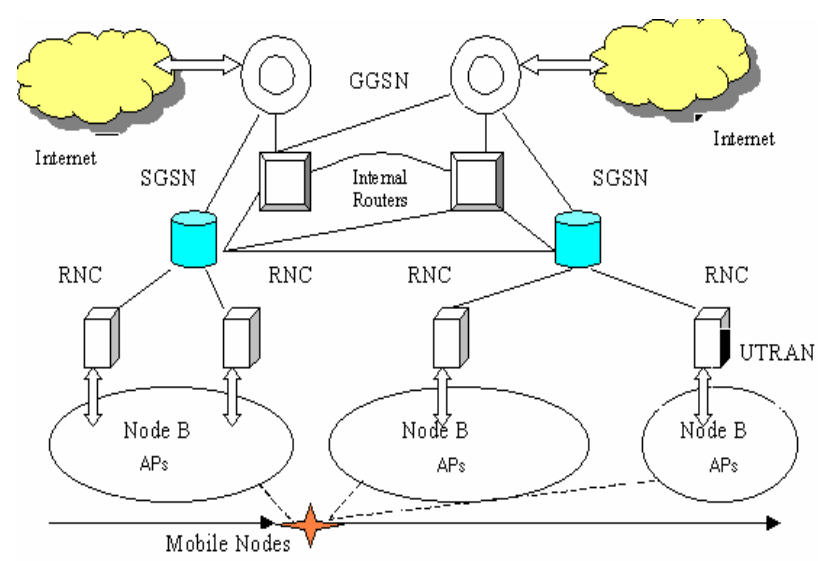

Fig 1. UMTS-GPRS Network

IP and MPLS Deployment [9], [10], [11]

There are two primary modes of IP deployment, Transport and Native. In the former the destination IP address of an end-user packet is not used to make forwarding decisions. Instead packets are encapsulated onto an intermediate layer, which may be specific to the wireless technology. These encapsulated packets are then transported over another IP layer. This allows for existing $2 \mathrm{G}$ components to remain untouched. In the latter case packets undergo regular forwarding without the case of any intermediate layer. Mobile networks employing this approach do not require nodes specific to any wireless technology. Further MPLS is a technology that used in conjunction with IP based systems can help in faster label lookup and switching. In an IP/MPLS [9] network the packet header is analyzed at the ingress to the network and is assigned to a particular FEC, it is encoded between layers 2 and 3 in the form of a label. At subsequent hops no IP header analysis is performed only the label is used as an index 
into the lookup tables. In addition to fast-forwarding MPLS offers other advantages such as; LSPs can be static or dynamically engineered to provide QoS guarantees. Traffic engineered LSPs can be provided with restoration paths for reliability.

\subsection{Overview [15],[16],[5],[8]}

The IEEE 802.11 standard accounts for ad-hoc and infrastructure modes. In infrastructure mode an AP performs point coordinate function by forming a cell in it's coverage area, called service set. A MN can associate with only one AP at a time. All the MNs connected to an AP communicate either via the AP or directly coordinated by the AP. Roaming across APs is supported in MAC. APs generate periodic beacons that advertise network ID (SSID) and cell ID (BSSID, AP MAC address). AT power up the MN sends an ASSOCIATE request to the AP. When MN moves to a different cell it receives a beacon with the same network ID but a different cell ID. It associates with the new AP by sending the MAC address of the old AP in the ASSOCIATE frame.

\section{INTEGRATION ARCHITECTURE}

One of the requirements of the architecture specification can be that the user in WLAN network can use the 802.11 accesses for UMTS PS service and the UMTS RNS for the UMTS CS service. One can maintain access to both connections in parallel but he cannot access the UTRAN for PS service. While the user in the UMTS network can use UMTS RNS for both PS and CS services. Once the MN moves into the 802.11 cells the PS connection via the UMTS domain is dismantled and is re-established through the WLAN network. Inside the WLAN the MN can use the UMTS RNS for CS service and thus it becomes mandatory for the MN to possess two distinct interfaces, one for the UMTS-GPRS and one for the 802.11 networks. We must understand that the radio specification for both 802.11 and UMTS-GPRS are different and it would require considerable effort to build an intercompatible system. Hence the integration point can certainly not be the RNC/3G-AR, a better logical choice would be to treat the SGSN (which is later modeled as a LEMA later in the paper) at the next highest level as the integration point since it is the next most closest to the wireless stack, see Fig. 2. Also the GGSN has not been chosen as the integration point because at the time of handover to UMTS the SGSN needs to recreate mobility state and acquire session PDP and RAB context, these are information that GGSN does not have and hence it would be inefficient to choose it as the integration point. Here since the MN must have two interfaces therefore there has to be a layer of abstraction says IP Handler layer between the UMTS and 802.11 device drivers and the normal IP layers to extract device specific context information for both the interfaces. MPLS when used in conjunction with these services offers a QoS sensitive abstraction that allows us to bind both these systems together efficiently.
It reduces the number of re-registrations at the core network routers and SGSNs by simply modifying the FEC path details and thus avoids the overheads related to frequent update messages between the $\mathrm{MN}$ and the core network routers.

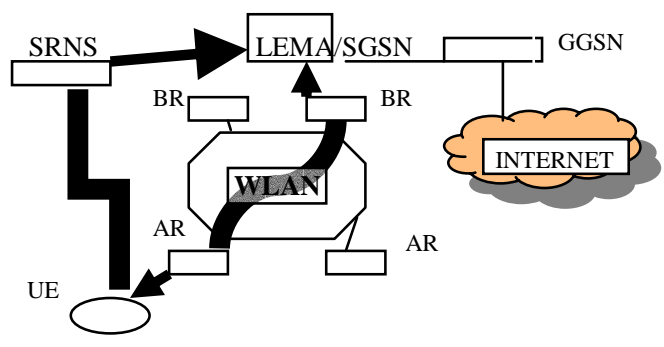

Fig 2. UMTS and 802.11 integration framework

Handoff Management of UMTS-WLAN networks

In GPRS mobility context related to mobile nodes mobility within UMTS is stored at both SGSN and MN, we can name it as GPRS mobility context. Since even in the 802.11 networks the GPRS connection is maintained hence the MN and SGSN keep the GPRS mobility context. The MN and the AR maintain mobility context information in WLAN. The GPRS mobility context does not contain any information related to mobility in WLAN. The WLAN APs periodically transmit beacons, which are received by all powered-up $\mathrm{MN}$ roaming within the AP coverage area; even those that are in power-saving mode. The MN detects it's move to WLAN by receiving beacons. It performs the UMTS-WLAN handover procedure, first the MN performs ASSOCIATION handshake with the AP, to establish layer 2 connectivity to the WLAN. The AP generates a signal for AR and gives a response to $\mathrm{MN}$. Signal causes AR to unicast RA (Router Advertisement) to MN. Now, layer 3 handover process starts, although the AR may not wait for router confirmation (RC) from $\mathrm{MN}$ before sending out $\mathrm{RA}$ thereby saving messaging overhead. Next the MN performs layer 3 handover. The RA contains important information about the AR such as it's IP address which is used by $\mathrm{MN}$ to perform Binding Update (BU), the BU contains SGSN address and other information as mobility context in the SGSN. When the AR receives the BU it authenticates the MN with the AAA server and provides the CoA (Care of Address). After authentication and CoA assignment the AR initiates handover procedure with SGSN by sending route-area-update message. Before sending the confirmation the SGSN sends a RAB-release message to the SRNC to release radio resources. When MN moves from WLAN to UMTS the interface detects missing beacons, and hence this procedure is termed as reactive handover, it attempts to detect AP by sending a probe packet. Failing to receive response to probe means handover from 802.11 to UMTS. MN sends route-areaupdate message to SGSN via it's UMTS interface, the message includes the address of the last AR it was connected to. The SGSN sends RESERVATION-TEAR 
message to release all resources previously held in WLAN network.

\section{MPLS Based Mobility Management of Proposed Integrated Architecture:}

The LEMA (Label Edge Mobility Agent) can act as an LER and as a general node in the $\mathrm{CN}$, and need not be placed at the edge only. It maps the destination IP of a packet with the respective FEC. As a MN moves from the scope of one AR to another within the scope of a given LEMA a signaling message sent to the latter causes the IP address of the MN to belong to a new FEC. The associated LSP will now point towards the new AR. The LEMA software is responsible for processing the mobility related signaling messages and dynamically changing the hosts IP address to a particular FEC, thus a LEMA can provide the service of a local HA to the MN. For widearea mobility the AR advertises a set of reachable LEMA's from it and also their topology. Registration at a particular level results in the hosts IP address being mapped to an LSP that points to the agent at the next lower level. The highest LEMA at which the MN is registered provides the $\mathrm{CoA}$ for ease in wide-area mobility. One of the main features of this scheme is that every MN has the flexibility to create it's own chain. Referring to Fig. 3, as MN moves from AR1 to AR3 it

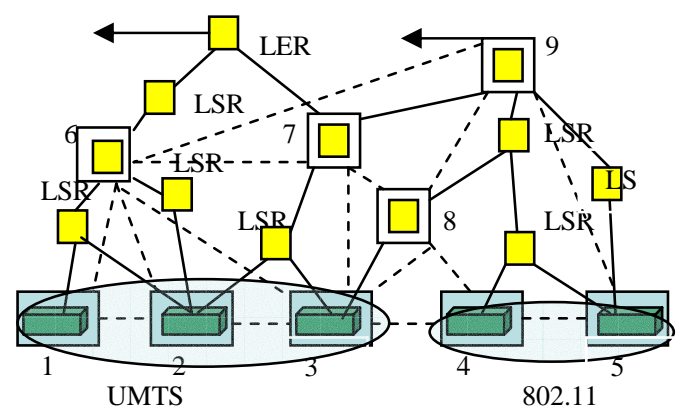

Fig 3. MPLS based UMTS and 802.11 LEMA integrated network

first registers with LEMA1, as it moves within scope of AR2 it registers with chain $(2,6,9)$ with node 9 serving as MIPv4 FA, movement from AR2 to AR3 also needs a single change in the chain $(3,6,9)$. Any change at the top level LEMA will result in MIP re-registration with hosts HA. The AR periodically sends advertisement messages such as subset of reachable LEMA's and their topology. The host receives $(1,(6,(7,(9))))$ rooted at the AR itself. After running the selection algorithm the MN chooses chain $(1,6)$ and registers with AR1 by specifying it's static ID and registers with LEMA6 by specifying ID of LEMA1. Now if MN moves out of scope of AR1 and receives $(2,(6,(7,(9))))$ all it needs to do is ask the most recent common node in this case LEMA6 to redirect the chain and thereby change the FEC mapping. As the host moves to AR3 it receives $(3,(8,(7,(9))))$ a comparison with current chain $(2,6)$ yields no match and thus host selects $(3,8,7)$ as the new chain and hence has to perform MIP reregistration at 7 as it was earlier rooted at 6 .

\section{Performance Analysis}

In this section the UMTS GPRS $3 \mathrm{G}$ architecture is compared vis-à-vis the proposed MPLS enabled integration architecture. The major consideration has to been to benchmark the performance of the traditional system with respect to the new architecture on the basis of various re-registration overheads involved. Whenever an MN moves outside the scope of the RNC that is currently serving that particular MN via the respective APs, the MN must re-register itself with the requisite network equipment in the $\mathrm{CN}$. This procedure deals with the $\mathrm{MN}$ signaling the FA and the HA to TEAR off the provisions made for that particular MN on the previous topological location. The MN re-registers itself with the FA in order to set up a new path to route packets intended for it towards its new destination. Location update to HA is carried out when the MN moves outside the scope of a particular clique of FAs. We have modeled the simulations on these principles. The hierarchical structure in the case of UMTS GPRS has been maintained using internal language constructs such as arrays and pointers to implement linked lists in standard ANSI C. The hierarchical topology of the traditional structure includes the GGSN at the very end of the communication domain, the SGSN at the next level and the 3G-AR or the 802.11 RNCs at the lower levels. The APs are placed at the bottom of the hierarchy. The data structure of the system has been modeled as an undirected graph. At each reregistration attempt standard UDP, 48 bytes header and 2 bytes of re-registration data have been incurred at each of the FAs or HAs. Considering the MPLS enabled proposed integration architecture we have also modeled the system as an undirected graph. However the number of reregistrations in this architecture has been found to be significantly lesser in comparison. To maintain a clear baseline we have used the same $48+2$ bytes packet format at each of the LEMAs in place of the SGSNs or the GGSNs. When the MN moves out of the range of a particular 3G-AR or 802.11 RNC then only the node that is lowest in the hierarchy and is common to both the old and new path incurs the overhead for the FEC redirection. New nodes on the changed path also incur the registration overhead for the first time only. There is no repeated reregistration charge at any common node between the old and new paths. The movement of the $\mathrm{MN}$ from one domain to another has been modeled as a random process using built in functions in standard ANSI C.

A performance comparison of the traditional UMTS GPRS architecture and the proposed MPLS enabled mobility friendly in terms of overheads is presented in Fig. 4. It is clear from the simulation results that the 
proposed MPLS enabled mechanism is more beneficial than the traditional UMTS GPRS architecture.

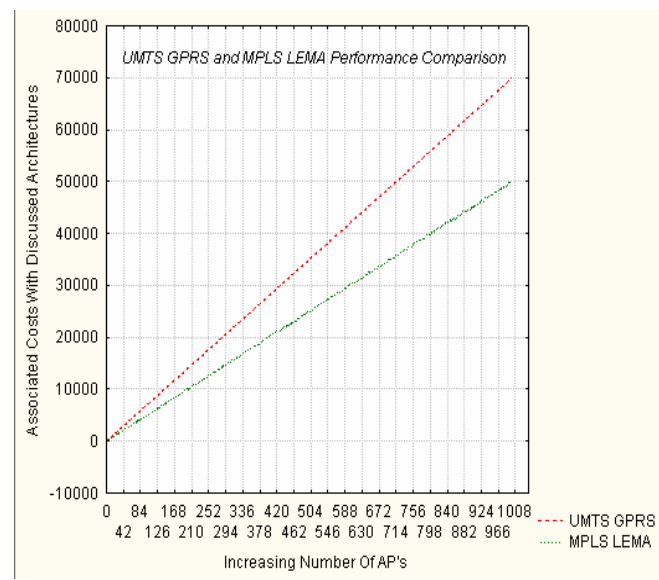

Fig 4. A performance comparison between UMTS GPRS Vs MPLS enabled scheme.

\section{Conclusion}

We have thus provided a classification of the ways in which IP with MPLS can be used to implement a mobile operator's infrastructure in $4 \mathrm{G}$ networks. Our proposed network model illustrates the benefits of using MPLS on the bedrock of Native IP coverage in order to extract efficiency form handover and mobility dependent networks. We have described the integration structure of 802.11 islands in the UMTS framework to provide seamless mobility from inter-AR to Inter-LEMA levels which allow for low overhead handover, simply changing of FEC mapping needed, and thus lead to efficiency in micro as well as wide area mobility transfers.

\section{REFERENCES}

[1] Andrew T. Campbell, "Untangling the Wireless Untangling the Wireless Web," Columbia University, CometGroup,http://www.Columbia.edu/itc/ee/e6951/2002 spring/lecturenotes.html, March 2002.

[2] Wee Seng Soh and Hyong S Kim, "QoS Provisioning in cellular networks based on mobility prediction techniques," IEEE Comm. Mag., 86-92, January 2003.

[3] Rudolf Roth, Mauro Campanella, Simon Leinen, et al., "IP QoS across multiple management domains: practical experiments from pan-European experiments," IEEE Comm. Mag., 62-69, January 2003.

[4] P.Demestichas, A.Taskaris, V.Stavroulaki, N.Mitrou and M.Thelogou, "Services and network level management strategies for third generation cellular systems," $4^{\text {th }}$ European Personal Mobile Communications Conference 2001Proceedings, February 20-22, 2001.

[5] Rod Walsh and Lin Xu, "Hybrid Networks: 4G World Using 3G Networks," Tampere University of Technology, http://www.cs.tul/fi/kurssit/8309700/seminars.html, March 2000.

[6] Hamid Aghvami, "A Vision for 4G," IEE Multimedia Communications launch event, http://www.iee.org/onComms/pn/multimediacomms/Aghv ami.pdf, December 2001.

[7] Upkar Varshney and Radhika Jain, "Issues in emerging 4G wireless networks," IEEE Computer, http://www.robinson.gsu/edu/facultyresearch/publication/ pubs2001/scholarly.html, 94-96, June 2001.

[8] NOKIA, "Whitepaper: Calling the Next Generation," www.nokia.com, April 2001.

[9] Fabio M Chiussi, Denis A Khotimsky and Santosh Krishnan, "Mobility management in third generation all IP networks," IEEE Comm. Mag., 124-134, September 2002.

[10] Fabio M Chiussi, Denis A Khotimsky and Santosh Krishnan, "A network architecture for MPLS based micro mobility," Proc. of IEEE Wireless Communication \& Networking, WCNCO2, Orlando, March 2002.

[11] Anwar Elwalid, Cheng Jin, Steven Low and Indra Widjaja, "MATE: MPLS Adaptive Traffic Engineering," IEEE INFOCOM 2001, 2001.

[12] Radu Dragos, Sanda Dragos and Martin Collier, "Design and implementation of an MPLS based load balancing architecture for Web switching," Proc. of $15^{\text {th }}$ ITC specialist seminar, Wurzburg, Germany, July 2002.

[13] Bin Wang and Jennifer C Hou, "Multicast routing and its QoS extension: Problems, algorithms and protocols," IEEE Network, 22-35, January/February 2000.

[14] Matthew Bocci and Jim Guillet, "ATM in MPLSbased converged core data networks," IEEE Comm. Mag., 139-145, January 2003.

[15] Muhammad Jaseemuddin, "An architecture for integrating UMTS and 802.11 WLAN networks," IEEE Symposium of Computer and Communication ISCC2003, Antalya, Turkey, Jun 30-July 3, 2003. 